Cell Research(1993),3, 165-171

\title{
The characteristics of the spindle fiber attachments (SFAs) enriched fraction from mouse nuclei ${ }^{1}$
}

\author{
NI ZumeI ${ }^{2}$, NING YI, YAN ZHOU, LUJI SHI(L. C. \\ $\mathrm{SZE})^{2}$. \\ Shanghai Institute of Cell Biology, Academia Sinica, 320 \\ Yoyang Road, Shanghai 200031, China.
}

\begin{abstract}
The characteristics of the particulate mouse centromere enriched fraction from isolated nuclei obtained in our laboratory were investigated by indirect immunofluorescence, test of the activity of microtubule organizing center(MTOC), SDS-PAGE, and fluorescence in situ hybridization. Most of the particles of the fraction are complexes of DNA and kinetochore proteins and show MTOC activity. The DNA isolated from the fraction can hybridize with DNA in the regions of the primary constrictions of all chromosomes of ascites cells. The kinetochore proteins isolated from the fraction are mainly those with molecular weight of $55 \mathrm{KD}$ and $59 \mathrm{KD}$. Results suggested that the fraction obtained is a centromere enriched nuclear fraction as indicated in our previous report
\end{abstract}

Key words: spindle fiber attachments ( SFAs), SFADNA, kinetochore proteins.

\section{INTRODUCTION}

Spindle fiber attachments (SFAs) or centromeres are chromosomal organelles which are necessary for regular anaphase separation of chromatids or homologous chromosomes from each other in meiosis or in mitosis respectively. Recent investigations on them have achieved a great progress. It has now become possible to define

1. The work is supported by China National "863 Project" for Biotechnology Development

2. To whom correspondence should be addressed. 
them more clearly as the structures formed by specific segments or sequences of chromosome DNA and their specifically bound proteins, the kinetochore proteins. As revealed by immunofluorescence studies with human CREST anticentromeric sera[1], they maintain their structure identity throughout the cell cycle. At metaphase of normal cell division they attach to or organize kinetochore microtubules. In cell free system supplemented with tubulin, they show the activity of microtubule organizing center.

The characteristics of SFAs or centromeres can be determined by methods now available. In short, their bound proteins, the kinetochore proteins, can be identified by human CREST anticentromeric sera, while their DNA, by either its specifically bound kinetochore proteins or its specific sites, namely the primary constrictions of the metaphasic chromosomes. The MTOC activity can be tested by incubating them with tubulin solution. Previously we have reported that mouse SFAs can be enriched greatly by a procedure consisting of treatment with concentrated salt solution, shearing with sonication, and centrifugation from interphasic nuclei[2]. By this procedure a particle preparation is obtained. In this report, the characteristics of the particles in the preparation determined by the above mentioned methods are given.

\section{MATERIAL AND METHODS}

\section{SFA enrichment method}

The nuclei of Ehrlich ascites cells were isolated by conventional method. The SFA-enriched nuclear fraction was prepared according to Shi et al.[2]

\section{Indirect immunofluorescence}

The SFA specific antiserum was obtained from human scleroderma CREST patients. Indirect immunofluorescence method according to $\mathrm{Ni}$ et al[3,4] was adopted for detecting the presence of SFAs. The SFA-enriched fraction was suspended in PBS followed by centrifuging the suspension onto slides. After fixation with PLP, the slides were processed for immunofluorescence observation. The slides were counter-stained with Hoechst 33258.

\section{Protein analysis and immunoblotting}

The proteins of SFA-enriched fraction were analyzed by SDS-PAGE according to Lamelli. The kinetochore proteins were detected by immunoblotting with SFA specific antiserum, the 2nd antibody used was peroxidase labelled goat-anti human IgG.

\section{Fluorescent In situ hybridization (FISH)}

The DNA isolated from the SFAs enriched fraction was biotin labelled with photobiotin according to Forster et al[5]. After labelling, DNA was store in $100 \%$ formamide until use. The cells were denatured in $70 \%$ formamide at $70^{\circ} \mathrm{C}$ for $3 \mathrm{~min}$ and labelled DNA was denatured in $100 \%$ formamide at $75^{\circ} \mathrm{C}$ for $10 \mathrm{~min}$ before use. The concentration of the labelled DNA used in the hybridization was approximately $5 \mu \mathrm{g} / \mathrm{ml}$ in $50 \%$ formamide, $10 \%$ dextran sulfate, $1 \%$ BSA, $2 \times$ SSC. Hybridization took place at $37^{\circ} \mathrm{C}$, for $18 \mathrm{~h}$. The slides were then washed successively in $50 \%$ formamide in $2 \times \mathrm{SSC}$ at $37^{\circ} \mathrm{C}$, in $2 \times \mathrm{SSC}$ at $37^{\circ} \mathrm{C}$, and in $2 \times \mathrm{SSC}$ at room temperature. They were stained with avidin conjugated to FITC in $4 \times$ SSC and FITC anti-avidin. 


\section{Test for the activity of microtubule organizing center (MTOC test)}

The particles of SFA-enriched fraction was suspended in a $2 \mathrm{mg} / \mathrm{ml}$ tubulin solution in assembly buffer. The mixture was then incubated at $37^{\circ} \mathrm{C}$ for $2 \mathrm{~h}$. At the end of incubation, the mixture was processed for electron microscopy. Alcoholic phosphotungstic acid was used for staining.

Tubulin was prepared according to Rosenbaum[6]. The tubulin preparation used in the present investigation was obtained from 3 cycles of polymerization depolymerization, without further purification.

\section{RESULTS}

\section{Indirect immunofluorescence observation with specific SFAs antiserum}

Previous works from various laboratories showed that human CREST serum can specifically decorate the SFAs or centromeres. The presence of SFAs or centromeres can be detected by this specific antiserum. Thus, the preparation of enriched SFAs was centrifuged onto slides with cytospin and processed for indirect immunofluorescence with the specific serum for obervation. In preparing the control slides, PBS was used instead of specific antiserum. Hoechst 33258 was employed as counter stain. The result was shown in Fig 1. In the experimental slides numerous fluorescent specks appeared under fluorescent microscope while only a few such specks in the control slides. Moreover, many particles which were decorated by specific SFAs antiserum were simultaneously stained by Hoechst 33258 known as DNA specific dye, Fig 2.

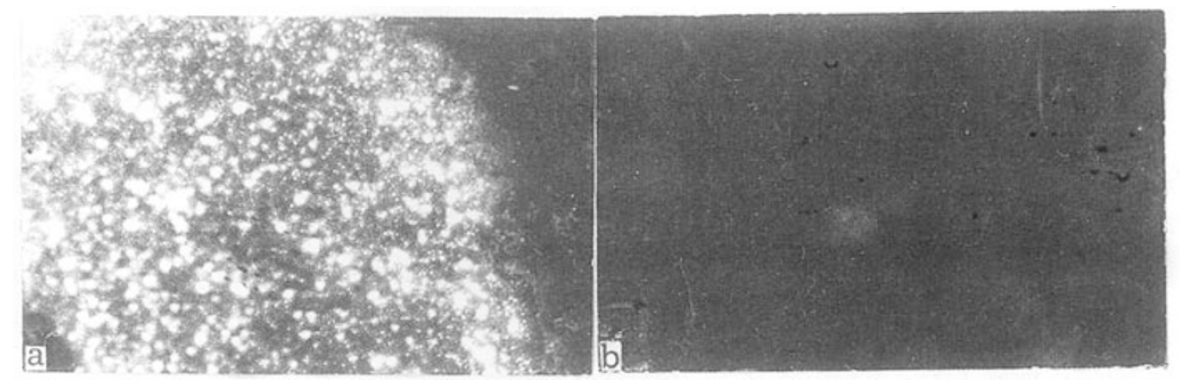

Fig 1. Indirect immunofluorescent staining of the particles of the SFA enriched fraction. a) shows that the particles are decorated by CREST anti SFA serum, b) illustrates that no fluorescent specks can be seen when PBS is used instead of the anti SFA serum in the process of indirect immunoflourescent staining.

The activity of microtubule organizing center (MTOC) of the enriched SFAs

The SFAs of metaphase chromosomes in cell or in vitro can attach to or organize kinetochore microtubules. This phenomenon may be denoted as MTOC activity. Previouly, evidences have been found in our laboratory that nuclei devoid of nuclear membrance have MTOC activity[7]. The MTOC activity of the preparation of en- 
The characteristics of SFAs enriched fraction

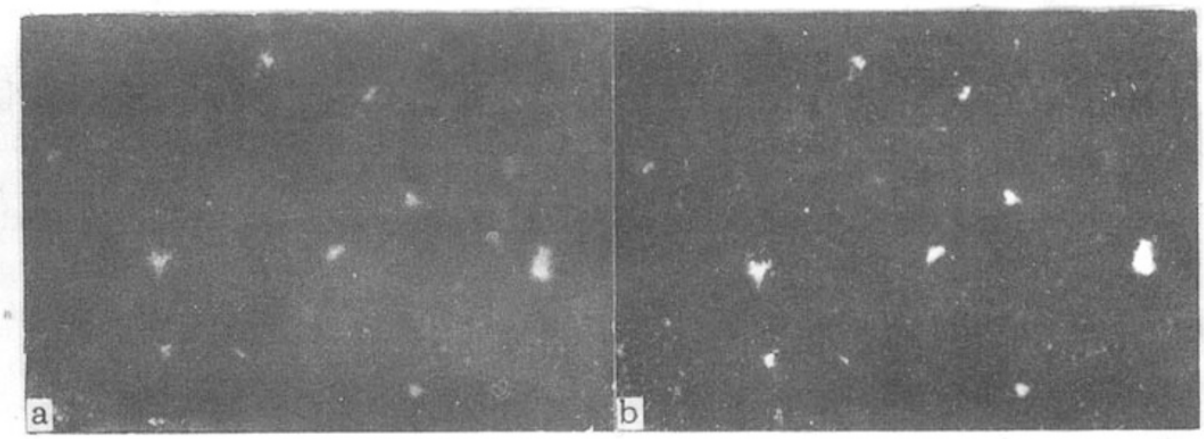

Fig 2. Indirect immunofluorescent staining with anti-SFA serum and counter staining with Hoechst 33258 of the diluted SFA-enriched fraction. The anti-SFA serum decorated particles in (a) are also stained by Hoechst 33258 in (b).

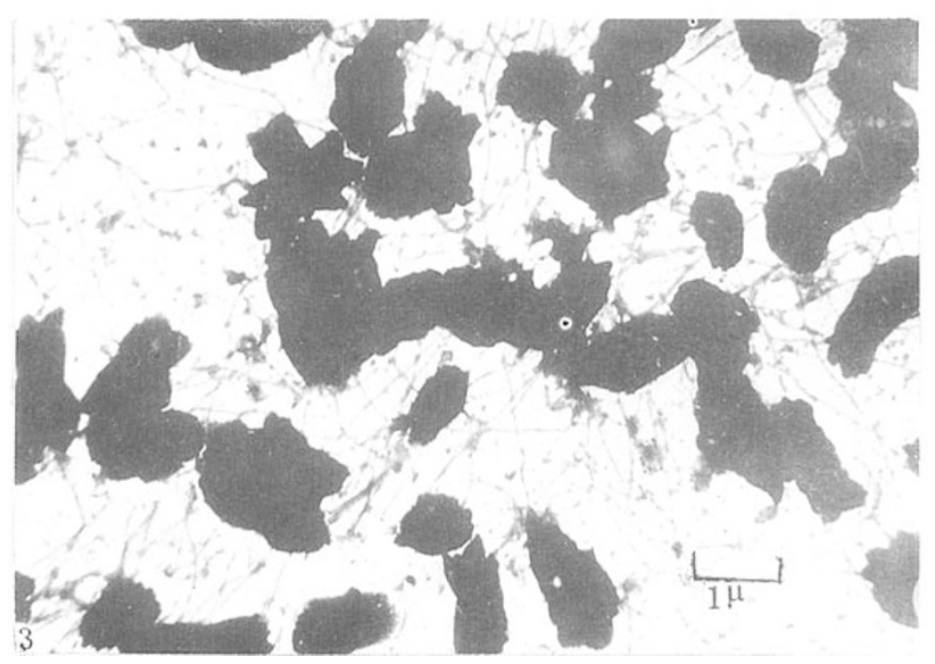

Fig 3. Electron micrograph shows that many particles associate with microtubules when the SFA-enriched fraction is incubated in tubulin solution.

riched SFAs was tested since it is derived from isolated nuclei. Thus, SFA-enriched nuclear fraction was incubated with tubulin. The result was shown in Fig 3 . It can be seen that many particles of the nuclear fraction obtained by our procedure associate with microtubules. This indicates that the preparation contains particles expressing MTOC activity.

\section{Proteins of the enriched SFAs}

The proteins of SFA-enriched nuclear fraction were analysed by, SDS PAGE. The result was shown in Fig 4. In comparison with whole nuclear extract, SFAs enriched fraction contained very little histones. This was expected when the high concentration of $\mathrm{NaCl}$ used in the procedure for obtaining the fraction was considered. 


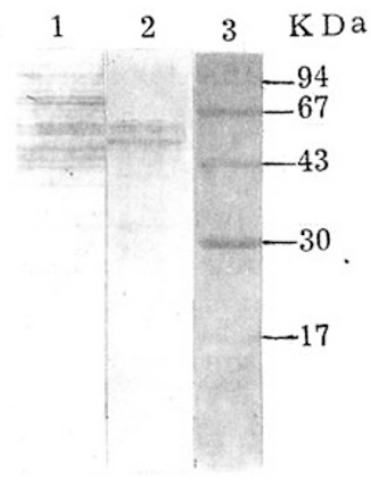

Fig 4. SDS PAGE of the proteins of the SFA enriched fraction. (1) proteins of the fraction. (2) immunoblotting of the protein with anti-SFA serum. molecular weight standard.

The proteins in the gel after SDS PAGE were transferred onto a sheet of nitrocellulose paper. Then the paper was immunoblotted with SFAs specific antiserum. The result was shown in Fig 4. The distinct protein bands were those with molecular weights of $55 \mathrm{KD}$ and $59 \mathrm{KD}$. The $59 \mathrm{KD}$ protein had been found to be localized in the primary constriction of mouse mitotic chromosomes[8].

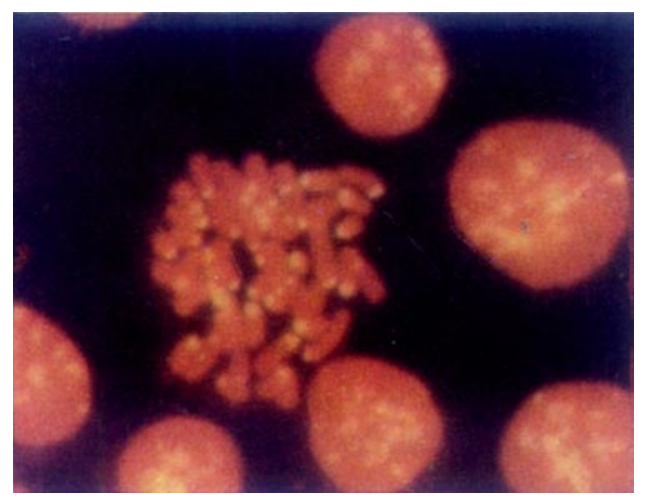

Fig 5. Fluorescent in situ hybridization of the DNA isolated from the SFAenriched fraction. The signal of the hybridization comes from the primary constrictions of all chromosomes of ascites cells

\section{The localization of the DNA isolated from the SFA-enriched fraction}

The DNA of the SFA-enriched nuclear fraction was isolated by SDS -proteinase $\mathrm{K}$ method. The DNA was then biotin labelled by the photobiotin method, and used for in situ hybridization. Fig 5 showed that, the labelled DNA from the mouse SFAenriched nuclear fraction hybridized with DNA situating in the primary constriction regions of all mitotic chromosomes of ascites cells. No hybridization signal from other regions of the mitotic metaphase chromosomes was detectable. Therefore, the DNA obtained was from the primary constriction regions of mitotic metaphase chro- 
The characteristics of SFAs enriched fraction

mosomes. It can be seen from the figure that in interphasic nuclei the DNA isolated distributed itself in the form of scattered small dots of varying size.

\section{DISCUSSION}

The results obtained from the present investigation indicated that the particulate preparation isolated from mouse interphasic nuclei by our procedures[2] was truly a nuclear fraction of enriched SFAs or centromeres. The main lines of evidence are summarized as follows.

(1) Most of the particles of the enriched preparation can be decorated by human CREST specific anticentromeric sera. These particles are also stained by DNA specific dye, Hoechst 33258, simultaneously.

(2) Many particles of the preparation can attach to or organize microtubules when the preparation is incubated with tubulin solution.

(3) The proteins of the preparation contain kinetochore proteins.

(4) The DNA of the preparation hybridizes solely with DNA in the primary constriction region of all metaphase chromosomes of ascites cells.

Since the procedures used in the preparation of the enriched SFAs is quite similar to that of preparing the nuclear matrix, the protein of the SFA-enriched fraction should be similar to some protein component of nuclear matrix. In the SFA-enriched fraction of mouse interphasic nuclei, the main kinetochore proteins revealed by Western blot with CREST serum were those with molecular weights, $55 \mathrm{KD}$ and $59 \mathrm{KD}$. The $59 \mathrm{KD}$ had been found in mitotic chromosomes of mouse fibroblasts. The presence of these in our preparation might mean that they were localized protein components of nuclear matrix in ascites cells. The localities of these proteins could serve as the binding sites for the SFA-DNA.

The demonstration of MTOC activity of many particles in SFA-enriched fraction raised some concern about the specific kinetochore protein which could be linked to microtubules. In Hela cells, evidences had been found suggesting that CENPB could bind to MAPs and function to link the microtubules to the kinetochore[9]. However, the main kinetochore proteins found in the SFA-enriched preparation were $55 \mathrm{KD}$ and $59 \mathrm{KD}$ proteins. No distinct band of $80 \mathrm{KD}$ protein was detected. Yet many particles in the enriched preparation showed MTOC activity. In mouse cells further research along this line is needed.

The DNA isolated from the preparation was found by in situ hybridization to be derived from the primary constriction region of all metaphase chromosomes of ascites cells. However, this finding did not necessarily mean that the isolated DNA consists entirely of the SFA DNA or centromeric DNA, since mouse satellite DNA was also located in the same region.

\section{REFERENCES}


[1] Moroi Y, Peebles C, Fritzler MJ, Steigerwald J, Tan EM. Autoantibody on to centromere (kinetochore) in scleroderma sera. Proc Natl Acad Sci USA 1980; 77:1627-31.

[2] Shi Luji (L. C. Sze), Ni Zumei, Lu Shifang, Wang Ge, Qi Huilin and Su Qingxiang. Enrichment of the spindle fiber attachments from mouse liver cell nuclei. Science in China (Series B), 1989; 32(7):816-21.

[3] Ni Zumei, Zhao Si and Shi Luji (L. C. Sze). The demonstration of fish spindle fiber attachments by indirect immunoflourescence with CREST anti centromere serum. KeXue TongBao, 1986; 31:1897-8.

[4] Ni Zumei, Shi Luji (L. C. Sze), Su Qingxiang and Su Lide. Indirect immunoflourescence studies on the spindle fiber attachments (SFAs) of several economical animals. Acta Zoologica Sinica, 1987; 33:297-9.

[5] Forster AC, McInnes JL, Skingle DC, Symons RH. Non-radioactive hybridization probes prepared by the chemical labelling of DNA and RNA with a novel reagent, photobiotin. Nucleic Acid Research. 1985; 13:745-61.

[6] Rosenbaum JL, Binder LI, Granett S, Dentler WL, Snell W, Sloboda R, Haimo L. Directionality and rate of assembly of chick brain tubulin onto pieces of neurotubules, flagellar axonemes and basal bodies. Ann N Y Acad Sci 1975; 253:147-77.

[7] Yan Yuanchang, Shi Luji (L. C. Sze). The formation of microtubules by the chromatin of the isolated nuclei with broken nuclear membrane. KeXue TongBao, 1984; 29:100-3.

[8] Hadlaczky G, Praznovszky T, Rasko I, Kereso J. Centromere proteins, I. Mitosis specific centromere antigen recognized by anti-centromere autoantibodies. Chromosoma (Berl) 1989; 97:2828.

[9] Brinkley BR. Centromeres and kinetochores: integrated domains on eukaryotic chromosomes. Current opinion in Cell Biology 1990; 2:446-52.

Received 10-4-1993. Revised 31-5-1993. Accepted 1-6-1993. 